\title{
The meaning of accelerated motion
}

\author{
Qing Li \\ ShiJiaZhuang Traditional Chinese Medical Hospital
}

\begin{abstract}
Unlike accelerated motion or curvalinear motion,a nonlinear motion state(non-inertial system) can be described by differential equations or the other algebric equations in axiom 2 , the accelerated motion in axiom 3 can be considered to be described by using the equation of the linear and curvilinear process of the continuum extending to infinitely distance.Further, this linear and curvilinear process of the continuum is a quantitative continuum in essence as a unity of infinitely quantities and infinitely dimensions at infinite distance (infinity) relative to all orientations in which we exist. These indicates that an accelerated motion accumulates continuously started from a finite quantities, leaping to transit from finite to infinite quantities by an infinitely great accelerating force.
\end{abstract}

Key words: the linear and curvilinear process of the continuum,one quantitative continuumm, the infinitely great, accelerating force

\section{MSC code: $51-X X$}

\section{Preliminaries}

1.1 The meaning of acceleration in axiom 2

Space and time are interrelated in axiom 2 [1] and the uniform linear motion(inertial system) is a special state of this relation here.However,in most cases, the relation between space and time is accelerated motion or curvalinear motion,a nonlinear motion state(non-inertial system), which is more complex motion state compared with uniform linear motion.Before describing the characteristics of nonlinear motion in axiom 1 and 3, the characteristics of accelerated coordinate system in common sense in axiom 2 will be illustrated in detail.

As mentioned earlier,the space and time is a continuum that does not have the largerest and smallest quantities but can be compared in sizes in which an given length can be arbitrarily and infinitely divided into smaller quantities. Any curve forms is meaningful in axiom 2 . There are three conditions for describing the properties of accelerated motion in non-inertial system.

1.1.1 The Cartesian coordinate system with vertical space and horizontal time is suitable for describing the physical quantity of accelerated motion in this axiom 2. Let $\mathrm{K}$ be the cartesian coordinate system at stationary state, and $\mathrm{K}$ 'be the Cartesian coordinate system with respect to $\mathrm{K}$ uniformly moving in a straight line with velocity v. An accelerated motion curve $\int$ (nonlinear curvilinear motion) being given in $\mathrm{K}$, as shown in Figure 1, will be given as $\mathrm{v} \int$ in $\mathrm{K}$ '. Here $\mathrm{v}$ is a constant, and $\int$ is a curve that can arbitrarily change shape $\left(\int\right.$ can have different shapes between $\mathrm{K}$ and $\mathrm{K}^{\prime}$ ). The definition of tangents in calculus, differential equations and partial differential equations can be applicable to describe the properties of $\int$. 
1

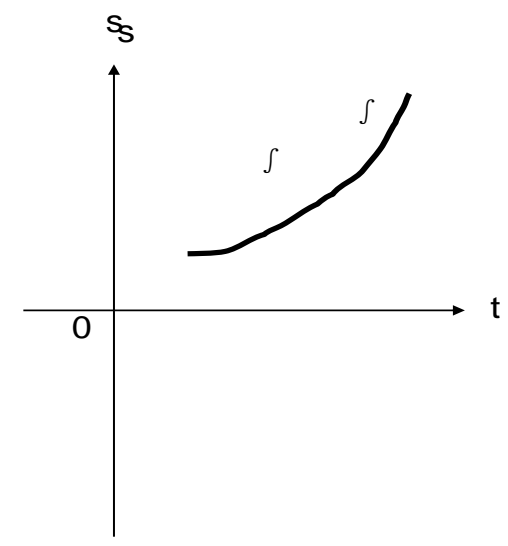

2

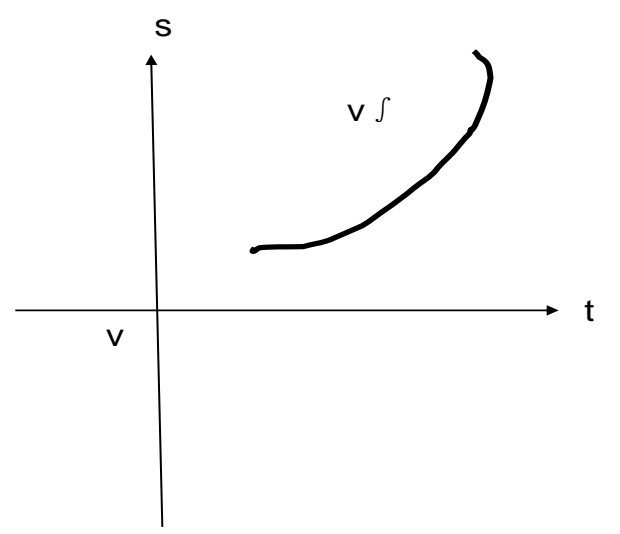

Figure $1 \mathrm{~K}$ ' is the Cartesian coordinate system with respect to $\mathrm{K}$ uniformly moving in a straight line with velocity $\mathrm{v}$. An accelerated motion curve $\int$ (nonlinear curvilinear motion) being given in $\mathrm{K}$ (1), will be given as $\mathrm{v}$ $\int$ in $\mathrm{K}$ '(2). Here $\mathrm{v}$ is a constant, and $\int$ is a curve that can arbitrarily change shape( $\int$ can have different shapes between $\mathrm{K}$ and $\mathrm{K}$ ' ).

1.1.2 Let $\mathrm{K}$ be the cartesian coordinate system at stationary state, and $\mathrm{K}$ ' be the Cartesian coordinate system with respect to $\mathrm{K}$ accelerating, An accelerated motion curve $\int$ (nonlinear curvilinear motion) being given in $\mathrm{K}$, as shown in Figure 2, will be given as $\iint$ in K'. Here $\int$ is still a variable that can change the shape of the curve arbitrarily, but the number of $\int$ is finite, that is a finite variable frame of reference of accelerated motion ( $\mathrm{K}$ as the reference). 
1

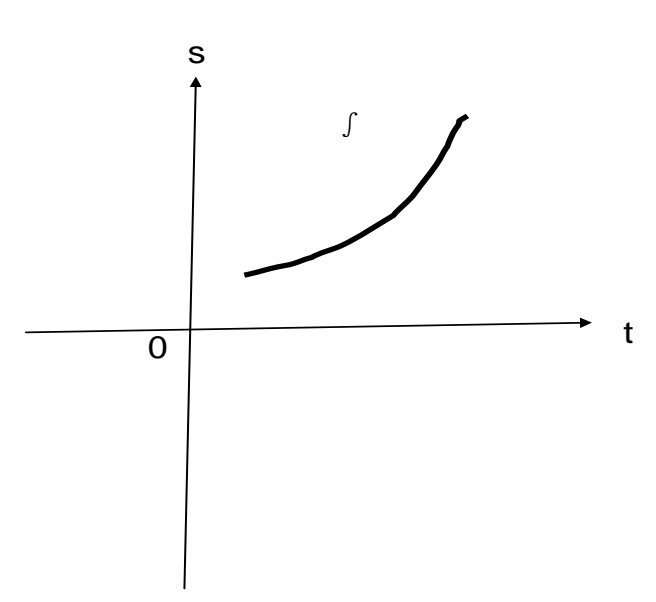

2

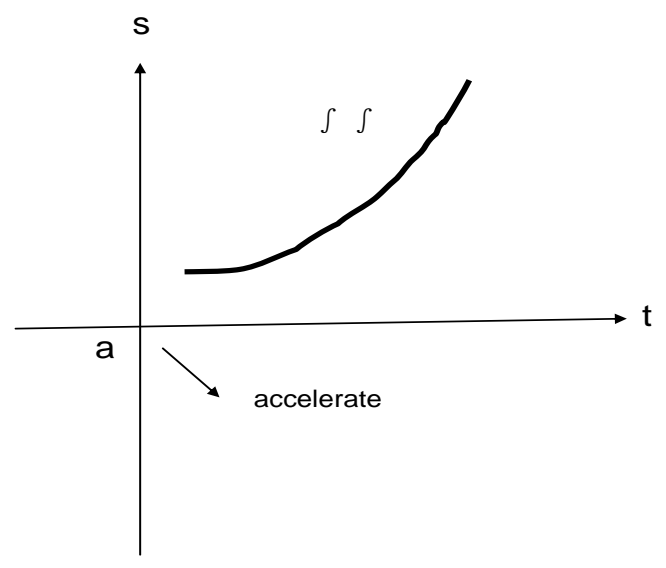

Figure $2 \mathrm{~K}$ ' is the Cartesian coordinate system with respect to $\mathrm{K}$ moving in accelerating velocity a.An accelerated motion curve $\int$ (nonlinear curvilinear motion) being given in $\mathrm{K}(1)$, will be given as $\iint$ in $\mathrm{K}^{\prime}(2)$. Here $\int$ is still a variable that can change the shape of the curve arbitrarily, but the number of $\int$ is finite, that is a finite variable frame of reference of accelerated motion .

1.1.3 The number of $\int$ is infinite, that is, an infinitely variable frame of reference for accelerating motion. $\mathrm{K}$ is meaningless as a frame of reference, a Cartesian frame of reference or some other finitely variable accelerated motion frame cannot describe this state.Instead of , A curve $\int$ belonged to a particular frame of reference cannot be given in this state. For any $\int$, it's a member of all states, and it is interleaved distribution with other curves that belong to all states. The state must be described by an infinite number of variables.

1.2 The meaning of acceleration in axiom 1

As mentioned earlier, space and time are independent of each other in Axiom 1[1,2]. For space or time, there exists an infinitely large and infinitely small quantity.This infinitesimal is zero point that can not be undivided into smaller parts where space and time are non-continuum composed of an infinite number of such zero points. Every given part can't be divided indefinitely or arbitrarily, it can only be divided finitely. The decimal point doesn't exist and the proportional extension of two different quantities can only be carried out in the form of unit () 1. In axiom 1, there is no two dimensional velocity, only single dimensional space and time. Unlike axiom 2, where the space and time measurements are expressed as multi-dimensional ratios, in Axiom 1, the space and time measurements are expressed as single-dimensional unit sizes. As mentioned earlier, uniform linear motion in Axiom 1 is carried out in units of 0 (such as 1,2, 3, 4, 5, and so on ). Now let's look at how accelerators are defined in Axiom 1 As shown in Figure 3-4. Note that the following number is unit values of 0 (integer multiples of 0 ), and the number interval of each line represents each step of the acceleration. The difference between each line and each line represents the difference in the unit value of the acceleration. 


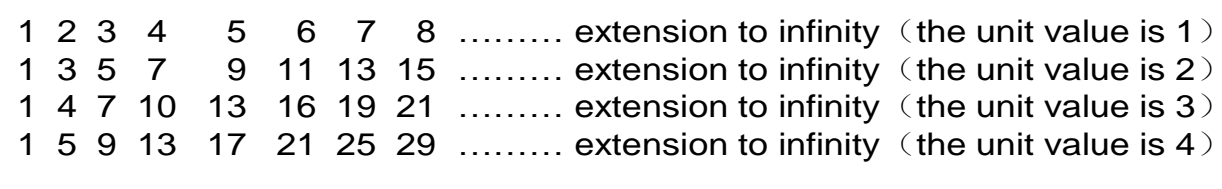

Figure 3 The uniformly accelerated motion in Axiom 1 (continuely change of velocity). The minimum acceleration force in Axiom 1 is given in line 1 .

$\begin{array}{cccccccccc}1 & 3 & 7 & 13 & 21 & 31 & 43 & 57 & \ldots \ldots \ldots \text { extension to infinity (the unit value is 1) } \\ 1 & 4 & 11 & 22 & 37 & 56 & 79 & 106 & \ldots \ldots \ldots \text { extension to infinity (the unit value is 2) } \\ 1 & 5 & 15 & 31 & 53 & 81 & 115 & 155 & \ldots \ldots \ldots \text { extension to infinity (the unit value is 3) } \\ 1 & 6 & 19 & 40 & 69 & 106 & 151 & 204 & \ldots \ldots \ldots \text { extension to infinity (the unit value is } 4 \text { ) }\end{array}$

And so on (variable change accelerated motion, variable variable change accelerated motion,.........)

Figure 4 Variable accelerated motion in Axiom 1 (continuely change of acceleration).

In axiom 1, since the higher-dimensional (larger than one-dimensional) space involves the infinite state of the space, the one-dimensional finite quantities described above is extracted from the high-dimensional space and thus has a special dominant position. According to this special one-dimensional finite quantity, the specific distribution characteristics of the two-dimensional 
finite quantity in the infinite space are as follows:

Unlike the accelerated motion in Axiom 2, which can be described by a continuously varying non-one-dimensional curve, the accelerated motion in Axiom 1 is the discontinuous accelerated motion in multidimensional space. As shown in figure 3. Being different from axiom 2 in which the acceleration force of the continuum is given by the ratio of variables ,The accelerated motion in Axiom 1 is carried out in units superimposed. The minimum acceleration force in Axiom 1 is given in line 1 of Figure 3. Note that the accelerated motion of axiom 2 and axiom 1 is different in the high-dimensional motion space. The former can be a continuous motion curve, while the latter can only be a broken line motion, that is, the former is a continuous changing process of order, while the latter is a jumping or irregular motion. As shown in figure 5.

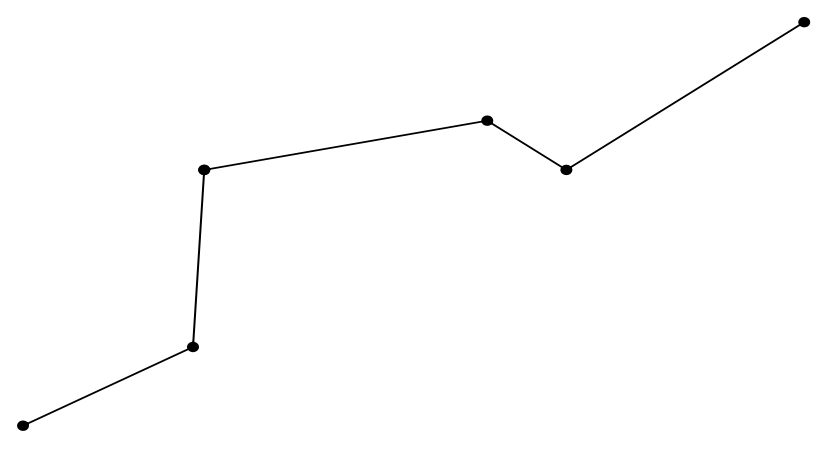

Figure 5 The accelerated motion is only a broken line motion on high-dimension space in axiom 1, that is, is not a continuous changing process of order, while it is a jumping or irregular motion .Each black spot indicates the unit values of 0 (integer multiples of 0 ) in two-dimension in this figure.

\section{Theorem}

2.1 The definition of acceleration in axiom 3

In axiom 3[3], infinitesimal is a one-dimensional finite quantities without sizes, and its sequential extension can only stay in a finite range. Only this infinitesimal can accomplish the accumulations or superpositions of infinite quantities by means of a change of direction, that is, to reach infinitely great quantities(infinitely many of finite quantities). So far a change in direction that represents an accumulations of infinitely many means a leap from one finite to an infinite quantities of finite. More importantly, the change in direction means that the finite quantities is not components of an infinite quantities. An infinite mamy quantities indicated by the change of direction exist in a quantitative continuum manner. The exact meaning of this a quantitative continuum is that it is connected to one another as a whole at infinite distance (infinitely great) relative to any orientation 
(all orientations) in which we exist. Here any orientation (all orientations)of our existence is indicated accurately as infinity of space whose parts are connected each other as a unity at the infinite distance (open ,unlimited space) [4].

2.2 The property of axiom 3 determines that there are two states, finite and infinite, for the equally proportional extension of two different quantities. In addition to these two states, they have no intermediate states, and in this sense it is different from the existence of multiple states in Axiom 2 (described earlier in Axiom 2). In a finite state, the relations between two finite quantities remain the same as the extension continues. In an infinite state, two quantities are infinite versus finite relations (an infinite unit value is infinite great quantities). Since a finite quantities is not an infinite quantities of components, a finite quantities stays in a finite range and the extension does only in an infinite quantities, as shown in Figure 6. The characteristic of infinite quantities indicates that its extension can not be continuously extended as do in extension between the finite quantities, that is, the infinite quantities can not continue to extend, we call this infinite extension as being not extending. In contrast to the invariance of two relations of finite quantities extension, we can also call this non-extensibility as permanent change. This permanent change may also be precisely defined as an infinite quantity which, as opposed to a finite quantity, cannot be extended, having the characteristic of perpetual change. This permanent change is equivalent to axiom 2 describing the third point of accelerated motion (a reference frame of infinitely variable accelerated motion). This permanent change can be described by the linear and curvilinear process of the continuum extending to infinitely distance. See figure 6.
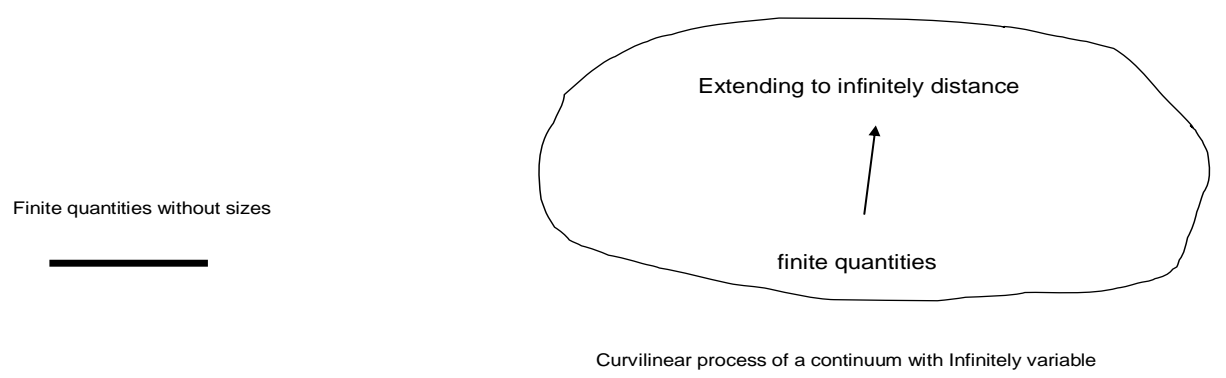

Figure 6 Since a finite quantities is not an infinite quantities of components, a finite quantities stays in a finite range and the extension does only in an infinite quantities, the infinite quantities indicated by the change of direction can not continue to extend, we call this infinite extension as being not extending. We can also call this non-extensibility as permanent change This permanent change can be described by the linear and curvilinear process of the continuum extending to infinitely distance.. 
Since this infinite quantities is the result of a change in direction, which also means that this infinite quantities exists as a quantitative continuum, so this conclusion is meaningless.

2.3 The above characteristics are the properties of accelerated motion and curvilinear motion described in axiom 3 with a single dimension, and the degree of change is used to represent the degree and complex characteristics of such motion. In Axiom 2, we usually use equations and formulas to describe the properties of some spatial curves. In Cartesian coordinates (space is ordinate, time is abscissa), the velocity $\mathrm{v}=\mathrm{s} / \mathrm{t}$ of uniform linear motion is a straight line, uniformly accelerated motion is expressed as $\mathrm{a}=\mathrm{dV} / \mathrm{dt}$ (the velocity changes over time), variable accelerated motion as a '=da/dt, and so on. It can be seen that with the continuous increase of dimensions (variables) (from two dimensions to an infinite number of dimensions), a straight line becomes a curve, and the curvature keeps increasing, that is, the description of the curve changes becomes more and more complex, or it can be said that with the increase of the number of variables, the properties of the curve described become more and more complex. From this we conclude that there are two ways to describe the complex properties of curve motion, the degree of change of single dimension and the number of variables of multiple dimensions, which cannot be substituted for each other. For example, describing the acceleration of a three dimension cannot be converted to describing the change of velocity of a two dimension.

2.4 Now let's look at how the number of variables with multiple dimensions characterizes spatial acceleration and curved motion. Assuming $\gamma$ is a variable,the equation of the curve $\int$ is expressed as $\int=\gamma_{1} / \gamma_{2} / \gamma_{3} \cdots \cdots$ Up to an infinitely many variables(note that the equation does not multiply it out).Considering the infinite definition of axiom 3, this equation is replaced by a continuous linear and curvilinear process (describing a single dimensional permanently changing continuous linear and curvilinear process). Being different from the definition of a single dimension, this continuous line and curve process describing the number of variables in multiple dimensions has the following characteristics:

(1) Representing an infinitely many variables indicated by the change of direction. Since each variable can only describe a finite process, the order number of variables is infinite, Namely the number of variables can be written in a line to cover the whole paper,until the whole universal space.

(2) This continuous line and curve process indicates that the curve is an quantitative continuum and must extend to infinitely distance.Since an combinations of finite and infinite form of curves is meaningless, The phenomenon in Figure 1 does not occur in axiom 3.

(3),The expression form is a continuum line and curve process, which is is a new express form being different from the existing common any equations of addition, subtraction, multiplication and division. Assuming the continuum curve process is $\in$, and there is an relational express $\in=$ infinite quantity/finite quantity(seeing figure 7). All equations describing finite processes and infinite processes are incomplete due to their characteristics in which a division equation can multiply it out and become a multiplication equation. Ordinary differential equations and partial differential equations, for example, cannot describe the continuum linear and curvilinear process representing an infinite many variables. 
An accelerated motion curve in finite range

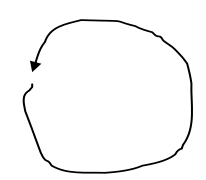

a

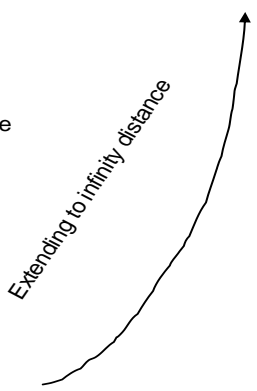

b

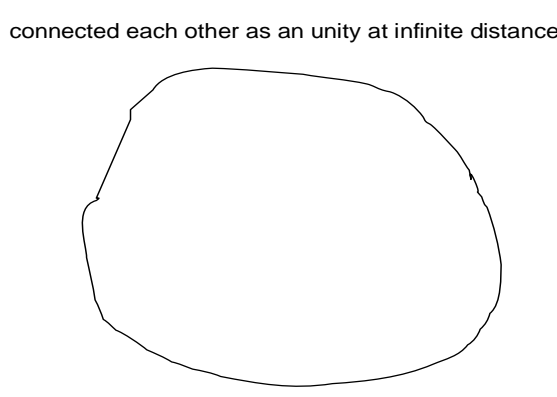

C

Figure 7. a. An accelerated motion curve in finite range. Since the higher-dimensional (larger than one-dimensional) space involves the infinite state of the space, this accelerated motion curve in finite range aslo relates to infinity. $b$. An accelerated motion curve of two dimensions extending to infinitely distance is a curvilinear process of the continuum . c. An unity of infinitely dimensions at infinite distance in which all its parts are connected each other as an unity at infinite distance, is aslo a linear and curvilinear process of the continuum .

Because of the single dimensional nature of axiom 1 and Axiom 3, it is meaningless to define multiple dimensions for acceleration and curvilinear motion. Accelerated motion can only be described by a single dimensional permanent change. Since the change of direction means the accumulation of an infinite many quanyities and the change of direction takes the form of a quantitative continuum, this so-called permanent change is aslo meaningless. Since a quantitative continuum in essence is an unity of infinitely quantities and infinitely dimensions at infinite distance, the terms 'the linear and curvilinear process of the continuum' is aslo meaningless, there is only the unity of a quantitative continuum to exist.

2.5 The difference between axiom 2 and Axiom 3 in describing accelerated motion and the meaning of acceleration in Axiom 3.

2.5.1 From the definition of axiom 2 we obtain a clear definition of its spatial geometry, which is summarized as follows.

(1) A high-dimensional space composed of points, lines, and planes. The measurement of space length gradually changes from small to large (the quantity of space can be compared).

(2) The space or time is a continuum, but a 0 point exists, as shown in Figure 8.

(3) There is no maximum or minimum value, that is, any quantity outside the system can be included within the scope of any given system of a certain length.

(4) The transition of dimension means the transition of infinite sizes, for example, the 2-dimensional finite area / 1-dimensional finite length $=\infty$ (representing infinite many of 
quantities), the 1-dimensional infinite length / 1-dimensional finite length $=\infty$, and the 1-dimensional finite length / 0 -dimensional 0 point $=\infty$. In other words, with the increase of dimensions, there is a expression form $\infty^{\infty}$ exists.

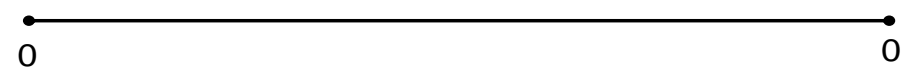

Figure 8 The space or time is a continuum, but a 0 point exists. This duality of space or time can be described that an zero point on an given length with property of continuum can not alter the sizes of this length .

From these four definition points we can see that an accelerated motion can be represented by a continuous curve, where accelerated motion is defined as non-linear or linear non-uniform motion. . Our current mathematical and physical descriptions of space and time are based on this state. For example, the tangent equation describing various shape curves, algebraic geometry equations are also based on this state. The two ways of describing the complexity of the curve's motion, the degree of change in a single dimension and the number of variables in multiple dimensions, are also interchangeable here, such as in our common differential equation (the division equation can also be multiplied to become the multiplication equation).

2.5.2 Now let's look at how axiom 3 redefines the properties of space geometry. In Axiom 3, space is a quantitative continuum without points, lines, planes, and higher dimensions, which we also generalize as follows.

(1) . The concepts of $0,1,2,3$, higher and infinite dimensions in axiom 2 do not exist, that is, the gradual increase of dimensions does not exist, such as the concept of curved space-time is meaningless. There is only one concept of infinite great quantity (infinite many quantity), which is an all-embracing infinite dimensions, and this infinite dimensions and infinite many quantities implicated by the change of direction are one concept.

(2) This infinite great is connected together as a unity at infinite distance (infinity) relative to any orientation (all orientations) in which we exist. In this case the exact meaning of any orientation is the infinity of space(infinite many quantities), and is components of the open, unrestricted infinite many quantities connected to one another at an infinite distance (open space). . 
(3) The unity as a whole is a quantitative continuum, indicating that there is only one quantity, and it is not composed of an infinite number of 0 points, but only one quantity.

(4) The change in direction represents the accumulation of an infinite many quantites, and the change in direction also means that we cannot establish any operation on this infinite great.

(5) A change in direction suggests that a finite quantity is not parts of this one-quantitative continuum, only existing as a reference quantity, and being an quantity without sizes.

2.5.3 The above five properties determine that the accelerated motion of axiom 3 has some new characteristics. If an accelerated motion accumulates continuously started from a finite quantities, it can only stay in a finite range, and there is no exact meaning of acceleration. This finite quantity can achieve the accumulation of an infinite number of its own quantities by means of the change of direction, reaching infinite great, then the concept of acceleration has been replaced by the concept of the so-called leap in Axiom 3 (the transition from finite to infinite). This infinity, like the unit accumulation in axiom 1, is the largest and unique unit quantity, that is, an infinite number of units, which we can also see as the infinite accelerating force.

\section{Prospective}

In my next paper, I will explain how these new features of space and time can be applied to our daily lives, and I will elaborate on the concepts of mass and energy.

\section{Declarations}

Availability of data and materials:

The [DATA TYPE] data used to support the findings of this study are included within the article.

\section{Competing interests:}

The authors declare no conflict of interest in preparing this article.

\section{Ethics statement:}

The study is informed consent for all authors in this manuscript.

\section{Funding:}

This research received no specific grant from any funding agency in the public, commercial, or not-for-profit sectors.

\section{Authors' contributions:}

Qing Li does full work in this manuscript.

\section{Acknowledgements:}

Not applicable 
Reference

1 Qing Li .,A non-continuum of the infinitesimal superposition. (vixra:2008.0067)

2 Qing Li, The meaning of an infinitely great velocity. (unpublish data)

3Qing Li, The meaning of the infinitely great (authorea:

DOI: 10.22541/au.160822935.50569408/v1)

4 Qing Li, The meaning of dimensional space (Zenodo: DOl:10.5281/zenodo.4381144)

\section{Authors' information:}

Qing Li

Affiliation : ShiJiaZhuang Traditional Chinese Medical Hospital

Code Number: 050051

No. 233 ,ZhongShan West Road

ShiJiaZhuang City

HeBei Province

PR. China.

Tel.: +86-13833450232

E-mail: liqingliyang@126.com

backup e-mail: 2958773632@qq.com

Author contributions state: Qing Li does full work in this manuscript.

Funding Acknowledgement: This research received no specific grant from any funding agency in the public, commercial, or not-for-profit sectors.

Conflict of interest statement: The authors declare no conflict of interest in preparing this article.

Qing Li,http://orcid.org/0000-0001-5049-123X

Academic email-address: jose.smith12802@campus.mccd.edu 
